\title{
A New Algorithm of Iris Location
}

\author{
Xiaomei Zheng ${ }^{\mathrm{a}^{*}}$ and Fang Zhao ${ }^{\mathrm{b}}$ \\ Zhengzhou Technical College, Zhengzhou450121, China \\ azztixiaomei@126.com, ${ }^{b}$ zhaojifang216@126.com
}

Keywords: Iris recognition; Iris location; Canny operator; Region detection; Least square method

\begin{abstract}
Aiming at the limitations of the existing iris localization algorithm, a new method of iris localization is proposed in this paper. The iris image is preprocessed firstly, the center coordinates and the radius of the iris inner edge is located by using Canny operator algorithm; the rough outer edge of the iris is got by the Canny operator secondly, and then the rough outer edge is divided into several small regions, the real edge of the outer edge of the iris can be got by using multi threshold segmentation in small regions. Because of the coupling relationship between the inner and outer edge of iris, the real center coordinates and the radius of the outer edge is located by the least square method. The experimental results show that this method can more quickly and accurately locate out the inner and outer edge of the iris.
\end{abstract}

\section{Introduction}

The visual appearance of the eye is composed of three parts, the scleral, the iris and the pupil. The scleral, the white part of the eye periphery, accounts for about $30 \%$ of the total area; the pupil, center of the eye, accounted for 5\%; the iris, located between the sclera and the pupil, contains the texture information, the most abundant occupy 65\%, the boundary of the iris and the pupil are approximately circular. Iris recognition technology is a technology based on the identification of biological characteristics, which has the advantages of life uniqueness, stability, be collected, and noninvasive and so on, it is the inevitable trend of the development of identity authentication in the research and application. Iris recognition technology is composed of image acquisition, iris location, feature extraction, iris coding, matching decision and so on. Iris location and feature extraction is the key of the iris recognition technology. The iris localization is the most critical step in iris recognition, which directly affects the feature extraction and recognition rate, which will lead to the failure of recognition algorithm.

Because the inner and outer edges of the iris are similar to the circle, the position of the iris can be regarded as a circle matching problem. Because the Hough transform (HT) is especially suitable for the detection of straight line, circle and ellipse shape [1], so it is often chosen as the matched filtering tools, the traditional iris localization is detecting the edge of the iris image and extracting the boundary, then Hough changes to the binary edge image; Jiewei Yu [2], Jing Zhao[3] et al implemented the iris localization based on the wavelet transform. Jingwei $\mathrm{Wu}$ [4] et al preprocesses the iris image to reduce the positioning of Hough transform computation and improve the positioning speed. A new method for iris localization proposed in the paper by the revelation of these methods.

\section{The Location of the Iris Inner Edge}

The eye image collected has the features of gray scale distribution, generally the pupil gray is less than the iris gray, the iris gray is less than the sclera grey, so the minimum grayscale area in the eye image is the pupil area. In order to enhance the gradient between the pupil and the iris, a grayscale transformation is applied to the eye image, the aim is to stretch the gray area of the image, the logarithmic transformation is adopted in this paper. Logarithmic transformation can make the image details of low gray level is more easy to see, can make the gray level distribution is even, and can match with the human visual characteristics also. The general expression for the logarithmic transformation is: 


$$
g(i, j)=a+\frac{\ln [f(i, j)+1]}{b * \ln c}
$$

$g(i, j)$ is the transformed image, $f(i, j)$ is the image before transform, $a, b, c$ are the parameters introduced to adjust the position and shape of the curve. The eye image before transform and the eye image after transform are shown in Fig. 1 and Fig. 2.

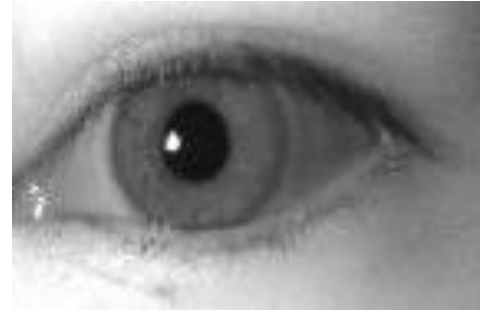

Figure 1. The original image

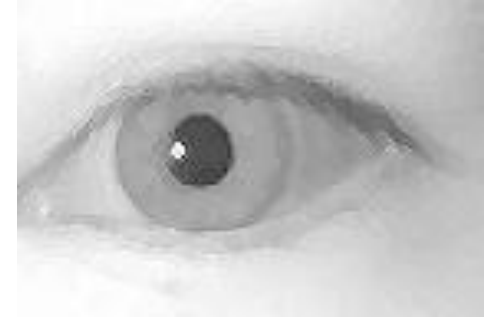

Figure 2. Image after logarithmic transformation

The gradient boundary of the iris inner circle is obviously increased from the transformed image, then the edge detection based on Canny operator is used to detect the image inner edge, Canny operator is based on the $\nabla G(x, y) * f(x, y)$, we get the edge strength and direction, and then detecting the inner edge by the threshold determination method.

Among:

$$
\begin{aligned}
& G(x, y)=\frac{1}{2 \pi \sigma^{2}} e^{-\frac{x^{2}+y^{2}}{2 \sigma^{2}}} \\
& \nabla G(x, y)=\left[\begin{array}{l}
\partial G(x, y) / \partial x \\
\partial G(x, y) / \partial y
\end{array}\right]
\end{aligned}
$$

And because the canny detection operator has three indexes: low error probability, high position precision, the detected edge should be on the real boundary; the only response to each edge is obtained. So the detection result of Canny operator is more close to the result of intuition.

The detection results show that a clear and complete inner edge of the iris can be obtained by chancing the appropriate threshold. After the global scanning on the image several times, the up, down, left, right tangent point on the inner edge of the iris can be obtained, so the inner edge of the iris center coordinates $x_{0}, y_{0}$ and the radius $r_{0}$ can be located.

\section{The Location of the Iris Outer Edge}

The location of the outer edge of iris is more difficult, the reason is the outer edge of the iris Influenced by the interference of noise, such as the upper and lower eyelids, eyelashes and so on, and the gradient between the iris and the sclera is not big is another reason. In order to suppress the noise, ensure internal continuity, the boundary obtained by Canny operator detection is not too much, a median filter method is used in two detection of Canny images firstly. Then the Canny operator detection is used to detect the edge of the smoothed image, the obvious boundary can be got by using the high threshold, and the boundary detected by the second Canny operator is as the rough outer edge of the iris.

Because the outer edge of the iris is rough, it just set up a broad scope, so the small area edge detection should be used, which is based on the results of the Canny operator detection, and divided the results into several small regions, accurately detected, and then the real edge of the outer edge of the iris can be got by using multi threshold segmentation in small regions.

Because there is a coupling relationship between the inner and outer edge of iris, so assuming that $x_{0}, y_{0}, r_{0}$ is the center coordinates and the radius of the inner edge, $x_{1}, y_{1}, r_{1}$ is the center coordinates and the radius of the outer edge, there is: 


$$
\sqrt{\left(x_{1}-x_{0}\right)^{2}+\left(y_{1}-y_{0}\right)^{2}}<d_{0}, \quad r_{1}-r_{0}<d_{r}
$$

Among them, $d_{0}$ and $d_{r}$ can be selected according to the size of the image and the size of the iris region. Here $d_{0}=6, d_{r}=25$ is selected.

According to the center coordinates and the radius of the inner edge of the iris detected, combined with $d_{0}=6, d_{r}=25$, and then the center coordinates of the outer edge of the iris can be located accurately by using the least square method. The least square method is: assuming that the point coordinates of the outer edge are $x(i), y(i), i=1 \ldots n$, the center coordinates and the radius of the outer edge are $x_{1}, y_{1}, r_{1}$, then:

$$
S=\sum_{i=1}^{n}\left[\left(x(i)-x_{1}\right)^{2}+\left(y(i)-y_{1}\right)^{2}-r_{1}^{2}\right]
$$

When $\mathrm{S}$ tends to the minimum, the $x_{1}, y_{1}, r_{1}$ is the real center coordinates and the radius of the outer edge, the location of the outer edge of the iris is realized.

\section{Experimental Results}

The original iris image used in the experiment was collected from the Japanese Panasonic iris recognition device. This algorithm is implemented on the computer of CPU 1.4GHZ, the programming environment is $\mathrm{VC}++6.0$.

In the experiment, firstly, the canny detection threshold value selected 70 , secondly the canny detection threshold value selected 20, and then the small regions detection is used in the most left point and the most right point of the rough location, the real outer edge point coordinates of the iris are obtained (only a part), which are shown in the Table 1:

Table 1 The real outer edge point coordinates of the iris (part)

\begin{tabular}{lllllllllllll}
\hline$i$ & $\mathbf{1}$ & $\mathbf{2}$ & $\mathbf{3}$ & $\mathbf{4}$ & $\mathbf{5}$ & $\mathbf{6}$ & $\mathbf{7}$ & $\mathbf{8}$ & $\mathbf{9}$ & $\mathbf{1 0}$ & $\mathbf{1 1}$ & $\mathbf{1 2}$ \\
\hline $\boldsymbol{x}_{\boldsymbol{i}}$ & 46 & 46 & 47 & 47 & 48 & 48 & 49 & 49 & 50 & 50 & 51 & 51 \\
$y_{i}$ & 28 & 68 & 28 & 68 & 28 & 68 & 28 & 69 & 27 & 69 & 27 & 69 \\
\hline & & & & & & & & & & & & \\
\hline$i$ & $\mathbf{1 3}$ & $\mathbf{1 4}$ & $\mathbf{1 5}$ & $\mathbf{1 6}$ & $\mathbf{1 7}$ & $\mathbf{1 8}$ & $\mathbf{1 9}$ & $\mathbf{2 0}$ & $\mathbf{2 1}$ & $\mathbf{2 2}$ & $\mathbf{2 3}$ & $\mathbf{2 4}$ \\
\hline $\boldsymbol{x}_{\boldsymbol{i}}$ & 52 & 52 & 53 & 53 & 54 & 54 & 55 & 55 & 56 & 56 & 57 & 57 \\
$y_{i}$ & 27 & 69 & 27 & 69 & 27 & 69 & 27 & 69 & 27 & 70 & 27 & 69 \\
\hline
\end{tabular}

According to the real coordinates of edge points in table 1, combining with Eq.4 and Eq.5, the center coordinates and the radius of the iris outer edge are obtained.

The results of the iris localization are as follows:

The center coordinates and the radius of the inner edge are $x_{0}=54, y_{0}=45, r_{0}=11$; The center coordinates and the radius of the outer edge are $x_{1}=56, y_{1}=45$, radius $r_{1}=26$. Fig. 3 shows the iris localization results.

\begin{tabular}{ccccccccc} 
Table 2 & \multicolumn{1}{c}{ The comparison between results of two algorithms } \\
\hline \multirow{2}{*}{ algorithm } & \multicolumn{1}{c}{ Inner edge parameters } & \multicolumn{4}{c}{ Outer edge parameters } & \multirow{2}{*}{ Time[s] } \\
& $x_{0}$ & $y_{0}$ & $r_{0}$ & $x_{1}$ & $y_{1}$ & $r_{1}$ & \\
HT & 55 & 45 & 10 & 56 & 46 & 25 & About0.12 \\
This article & 54 & 45 & 11 & 56 & 45 & 26 & About0.08 \\
\hline
\end{tabular}

Under the same conditions, the location results of the two algorithms and the times needed are showed in the Table 2, we can see the location results of the two algorithms is very close to the real edge, but the time that the algorithm proposed in this paper needed is shorter than the time that the 
Hough transform algorithm[5] needed.

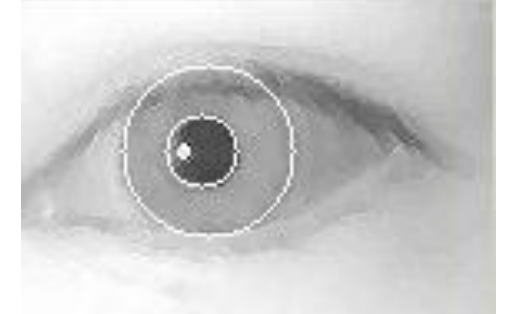

Figure 3. Iris location results

\section{Conclusions}

The experimental results show that using the Canny operator to detect the inner edge of the iris, global scanning on the iris to locate the center coordinates and the radius of the inner edge, then locate the outer edge of the iris roughly, using multi threshold values detection in small areas to locate the real outer edge points in the far-left area and the far-high area, which can reduce the computation amount to improve the location speed, Table 2 shows the time the iris location needed is reduced from $0.12 \mathrm{~s}$ to $0.08 \mathrm{~s}$. And the algorithm proposed in this paper is relatively simple, easily to implement, is a more effective method of iris location.

\section{References}

[1] W.W. Sun, M.X. Zhou and C.J. Bai: Computer \& Digital Engineering, Vol. 44 (2016) No.1, p.113. (In Chinese)

[2] J.W. Yu, Y.Z. Shen and X.H. Ji: Journal of Zhejiang University of Technology, Vol. 33(2005) No.2, p.181. (In Chinese)

[3] J. Zhao: Computer Technology and Development, Vol. 23(2013) No.4, p.51. (In Chinese)

[4] J.W. Wu and Q. Wu: Journal of Tianjin University of Technology, Vol. 21(2005) No.3, p.74. (In Chinese)

[5] G. Chen and Y.L. Zhou: Journal of East China University of Science and Technology, Vol. 30(2004) No.2, p.230. (In Chinese)

[6] Q.R. Li and Z. Ma: Journal of University of Electronic Science and Technology of China, Vol. 31(2002) No.1, p.7. (In Chinese)

[7] J.H. Lu and J.P. Sun: Electric Power Science and Engineering, Vol. 32(2016) No.8, p.65. (In Chinese)

[8] X.F. Diao: The Research of Fast Iris Localization Algorithm (MS., Lanzhou University of Technology, China 2008), p.20.

[9] G.M. Liang: Iris Localization Technology and Its Applications under Various Light Conditions (MS., Lanzhou University, China 2012), p.19.

[10]S.J. Miao: The Research on Segmentation Method of Human Iris Image (MS., National University of Defense Technology, China 2005), p.12. 